\title{
Ultrarelativistic electron beam spatial size estimation from angular distribution emission in thin crystals
}

\author{
Yu.A. Goponov ${ }^{\text {a }}$, M.A. Sidnin ${ }^{\text {a }}$, K. Sumitani ${ }^{\text {b }}$, Y. Takabayashi ${ }^{\text {b }}$, I.E. Vnukov ${ }^{\text {a,* }}$ \\ a Belgorod National Research University, Belgorod, Russia \\ b SAGA Light Source, 8-7 Yayoigaoka, Tosu, Saga 841-0005, Japan
}

\section{A R T I C L E I N F O}

\section{Article history:}

Received 23 June 2015

Received in revised form

9 November 2015

Accepted 9 November 2015

Available online 19 November 2015

Keywords:

Collider

Electron beam divergence

Beam size

Crystal

Diffracted transition radiation

Crystal structure destruction

\begin{abstract}
A B S T R A C T
The use of ultrarelativistic electron (positron) emission in thin crystals to estimate particle beam spatial sizes for projected electron-positron colliders is proposed. The existing position-sensitive X-ray range detectors restrict the minimum value of the measured beam size to a level of approximately $10 \mu \mathrm{m}$, which is far greater than the planned sizes of collider beams. We propose to estimate the electron (positron) beam divergence over the diffracted transition radiation from angular distribution measurements. The spatial size can be obtained from the calculated beam emittance or the experimental emittance, which is measured during the earlier stage of acceleration using optical transition or optical diffraction radiation. The problem of crystal destruction under the influence of a high-intensity electron beam is discussed. The use of surface parametric X-ray radiation, where the problem of crystal destruction is almost absent, to measure the electron beam parameters is also discussed.
\end{abstract}

(c) 2015 Published by Elsevier B.V.

\section{Introduction}

One of the most important parameters that determine the efficiency of projected electron-positron linear colliders $[1,2]$ is the luminosity:

$L=\frac{n_{b} N^{2} f_{r e p}}{4 \pi \sigma_{x} \sigma_{y}} H_{D}$

where $n_{b}$ is the number of bunches, $N$ is the bunch population, $f_{\text {rep }}$ is the repetition rate, $H_{D}$ is the luminosity enhancement factor, and $\sigma_{x}$ and $\sigma_{y}$ are the characteristic beam sizes in the horizontal and vertical directions, respectively. The estimated luminosity, $L$, of the International Linear Collider (ILC) is approximately $10^{34} \mathrm{~cm}^{-2} \mathrm{~s}^{-1}$ and higher, due to the small size of the beam at the interaction point of $\sim 700 \times 7 \mathrm{~nm}^{2}\left(\sigma_{x} \times \sigma_{y}\right)$ [1]. Invasive [3] and non-invasive $[4,5]$ methods developed to determine the transverse beam size based on the registration of optical radiation from metal foils set in the accelerator cannot ensure measurement of the beam parameters with such small sizes, due to coherent effects in the radiation [6].

One method that can provide non-invasive measurement of the spatial dimensions of an ultra-fast electron beam is to use the Shintake monitor, based on the interaction of electrons with a target of laser interference fringes, and record the scattered

\footnotetext{
* Corresponding author.

E-mail address: vnukov@bsu.edu.ru (I.E. Vnukov).
}

Compton photons emitted in the direction of the electron beam [7]. Recently, this method has been used to measure the dimensions of a beam (High Energy Accelerator Research OrganizationAccelerator Test Facility; KEK-ATF) in the range of $20 \mathrm{~nm}$ to several microns with an accuracy of no more than $10 \%$ [8]. To use this method, the electron beam must be bent by a magnet after interaction; therefore, its use under the conditions of the ILC would require significant additional expenditure. For the same reason it cannot be used in a collider regime where there are two simultaneous particle beams or for intermediate diagnostics and control of the beams inside the accelerator during the acceleration process.

Another way to address this issue could be a decrease of the detected radiation wavelength by switching to the X-ray frequency range and employing the mechanism of parametric X-ray radiation (PXR) proposed in Refs. $[9,10]$. The choice of radiation mechanism corresponds with the large angles of PXR emission in the direction of the electron motion and it can be detected relatively easily with conventional X-ray detectors.

In the first approximation, PXR may be considered as coherent scattering of the electromagnetic field of a particle on the electron shells of periodically arranged target atoms [11,12]. By analogy with X-ray diffraction in crystals, there are two approaches to the description of PXR. The kinematic approximation suggests that the multiple reflections of PXR photons at crystal planes are negligibly small. If this condition is not satisfied, then it is necessary to use dynamical theory. PXR can also be described as a coherent polarization bremsstrahlung of relativistic charged particles in a crystal 
[13] (see also [14]). As part of this approach, it has been shown [15] that for perfect crystals the contribution of dynamic effects is not more than $10 \%$; therefore, the kinematic approximation should be sufficient to describe the experimental data. Numerous experimental works (see, for example, [16] and references therein) have demonstrated that the kinematic PXR theory describes the results of measurements for electron energies from a few mega-electronvolts to several giga-electron-volts with an accuracy better than $10-15 \%$.

A study on the influence of the electron beam size on the PXR spatial distribution from $855 \mathrm{MeV}$ electrons in a $50 \mu \mathrm{m}$ thick silicon crystal using a high-resolution X-ray camera [17] has confirmed that estimation of the electron beam size is possible with such measurements [18]. Electron beam size measurements for an energy of $255 \mathrm{MeV}$ using the PXR spatial distribution in a $20 \mu \mathrm{m}$ thick silicon crystal made with a coordinate detector based on an imaging plate [19] coincided with those using optical transition radiation (OTR) [20].

For fast electrons, PXR is always accompanied by radiation diffracted in the crystal, which is generated directly inside the target or on its surface [22,21]. In the first case, diffracted bremsstrahlung (DB) is considered, while in the second case, diffracted transition radiation (DTR) is considered. The former is dominant under the condition $\omega \gg \gamma \omega_{p}$, where $\omega$ is the photon energy, $\gamma$ is the Lorentz factor, and $\omega_{p}$ is the plasma frequency of the medium, whereas the latter is dominant under the opposite condition. If the condition $\omega \sim \gamma \omega_{p}$ is true, then the contributions of both radiation mechanisms are observed. Taking into account the real photon diffraction for the $[18,23]$ experiments, an improved agreement between the experimental and calculated data was obtained for the center of PXR reflex [24], where a large difference between the experimental results and calculated ones without the contribution of real photons diffraction was observed $[18,23]$.

Unlike the Shintake monitor, a device that can realize this method of electron beam size measurement can be used at any stage of acceleration, requires significantly less expenditure, and can be relatively easily integrated into the accelerator control system by replacing the existing beam monitors based on OTR [3], optical diffraction radiation (ODR) [4], and Smith-Purcell radiation [5]. However the change in the ratio of PXR and diffracted real photons of TR and bremsstrahlung with an increase of electron energy up to $100-500 \mathrm{GeV}$ [25] and a decrease in the size of the beam has yet to be clarified. Previous measurements $[10,18]$ were performed for electron energies less than $1 \mathrm{GeV}$ and beam size is about hundred microns and greater.

Thus, the unresolved problems, yet obvious advantages, of using the X-ray emission of electrons in thin crystals to diagnose the parameters of ultra-high energy electron beams suggests that research in this area is important and relevant.

\section{Theoretical considerations}

In the experiment, all the radiation mechanisms generated at the Bragg angles are implemented simultaneously. The basic formulae and approaches for each mechanism that was used for calculations are introduced here, mainly following Refs. [21,24]. The kinematic PXR theory describes the results of measurements quite well; therefore, the PXR yield was calculated using a PXR spectral-angular distribution formula obtained in the kinematic approximation presented in Ref. [26]:

$$
\frac{d^{2} N}{d Z d \Omega}=\frac{\sum_{\alpha} \alpha \omega^{3}\left|\chi_{\vec{g}}\right|^{2}}{2 \pi \varepsilon_{0}^{3 / 2} \beta\left(1-\sqrt{\varepsilon_{0}} \vec{\beta} \vec{n}\right)}\left[\frac{(\omega \vec{\beta}-\vec{g}) \vec{e} \vec{k}_{\alpha}}{\left(\vec{k}_{\perp}+\vec{g}_{\perp}\right)^{2}+\frac{\omega^{2}}{\beta^{2}}\left\{\gamma^{-2}+\beta^{2}\left(1-\epsilon_{0}\right)\right\}}\right]^{2} .
$$

The system of units used is $\hbar=m_{e}=c=1$. Here, $\epsilon_{0}=1-\omega^{2} / \omega_{p}^{2}$, $\vec{\beta}=\beta \vec{n}_{0}$ is the electron velocity vector, $\vec{n}_{0}$ and $\vec{n}$ are the individual vectors that correspond to the incoming electron and emitted photon (with energy $\omega$ and momentum $\vec{k}$ ), respectively, $\vec{g}$ is the reciprocal lattice vector, $\vec{e} \vec{k}_{\alpha}$ are the polarization vectors, and $\perp$ is the index that denotes the vector projection on the plane normal to $\vec{n}_{0}$. The other notation is conventional. The term $|\chi \vec{g}|^{2}$ denotes the following value:

$\left|\chi_{\vec{g}}\right|^{2}=|S(\vec{g})|^{2} \exp (-2 W)\left[-\frac{\omega_{p}^{2}}{\omega^{2}} \frac{f(\vec{g})}{Z}\right]^{2}$.

In expression (3), $|S(\vec{g})|^{2}$ is the structure factor, $\exp (-2 W)$ is the Debye-Waller factor, and $f(\vec{g})$ is the Fourier component of the spatial distribution of electrons in a crystal atom $(f(0)=Z$, where $Z$ is the number of electrons in an atom).

The divergence of the electron beam incident on the target, the multiple scattering of the electrons in the crystal, collimation of the radiation, and other experimental factors were taken into account with respect to the procedure described in Ref. [27].

For high-energy electrons, the radiation in the X-ray range of photon energy $(\omega \leq 00 \mathrm{keV})$ in crystals, except PXR, is generated through the mechanism of the transition radiation TR. The bremsstrahlung intensity is suppressed due to the Ter-Mikaelian effect $[28,29]$.

The spectral-angular distribution of the TR intensity on the vacuum environment without taking into account the polarization of the radiation can be represented as [30]:

$\frac{d^{2} I_{T R}}{d \omega d \theta}=\frac{2 e^{2} \theta^{3}}{\pi}\left|\frac{1}{\theta^{2}+\gamma^{-2}}-\frac{1}{\theta^{2}+\gamma^{-2}+\omega_{p}^{2} / \omega^{2}}\right|^{2}$

where $\theta$ is the photon emission angle according to the direction of the electron motion. For simplicity, it can be assumed that the TR is generated directly at the inlet into the crystal and is then diffracted therein.

The angle of photon emission in expression (4) is measured from the direction of motion of the emitted electron, whereas it is generally measured experimentally according to the dependence of the resulting radiation yield $Y(\omega, \theta)$ in the collimator aperture $\vartheta_{c}$ on the observation angle $\theta$, with respect to the reflex center located at an angle $\Theta_{D}$.

To obtain such information, it is necessary to take into account the angular distribution of the electron beam incident on a crystal $P\left(\theta_{e}, \varphi_{e}\right)$. In this case, the spectral-angular distribution of $\mathrm{TR}$, $\frac{d^{2} I_{T R}^{*}(\omega, \theta, \varphi, t)}{d \omega d \Omega}$, is defined by a convolution of spectral-angular distribution of TR with the angular distribution of electrons, which can be expressed as:

$\frac{d^{2} I_{T R}^{*}(\omega, \theta, \varphi, t)}{d \omega d \Omega}=\int P\left(\theta_{e}, \varphi_{e}\right) d \Omega_{e} \int \frac{d^{2} I_{T R}\left(\omega, \theta_{\gamma}, \varphi_{\gamma}\right)}{d \omega d \Omega_{\gamma}} f\left(\vec{n}, \vec{n}_{e}, \vec{n}_{\gamma}\right) d \Omega_{\gamma}$.

Here, $\vec{n}_{e}\left(\theta_{e}, \varphi_{e}\right)$ and $\vec{n}_{\gamma}\left(\theta_{\gamma}, \varphi_{\gamma}\right)$ are vectors that describe the directions of electron and photon propagation in the laboratory frame of reference and in the system correlated with the direction of the electron motion, respectively. $\vec{n}(\theta, \varphi)=\vec{n}_{e}+\vec{n}_{\gamma}$ is a vector for the direction of the photon in the laboratory frame and $f(\vec{n}$, 
$\vec{n}_{e}, \vec{n}_{\gamma}$ ) is a function that describes the correlation between these vectors.

Therefore, the angular distribution of diffracted radiation relative to the reflex center along the axis $x$ (see, for example, Ref. [22]) can be represented as:

$Y_{D T R}\left(\omega, \theta_{x}\right)=\int d \omega \int \frac{d^{2} I_{T R}^{*}}{d \omega d \Omega} R\left(\omega, \vec{n}, \vec{g}, \Theta_{D}\right) S^{*}(\omega, \vec{n}, T) d \Omega$

where $R\left(\omega, \vec{n}, \vec{g}, \Theta_{D}\right)$ is the reflectivity for the directions represented by vectors $\vec{n}$ and $\vec{g}$, which is defined by the crystal orientation angle $\Theta$ and the location of the detector $\Theta_{D}$. $S^{*}(\omega, \vec{n}$, $T$ ) is a function that takes into account the photon absorption in the crystal and the geometry of the experimental set-up. The integration in (6) occurs over all angles and photon energies with the incidence of the photons on the collimator.

To determine the yield of the diffracted radiation, it is necessary to determine the reflectivity of the crystal, $R\left(\omega, \vec{n}, \vec{g}, \Theta_{D}\right)$. Here, the method described in Ref. [24] was used, which allows multiple Bragg re-scattering, absorption, and scattering photons due to processes that are not associated with diffraction to be taken into account.

\section{General considerations}

Using X-rays to measure the size of a beam of fast electrons by the PXR mechanism $[10,18,20]$ is restricted to the minimum size that can be measured with coordinate detectors of X-ray range. The spatial resolution of the devices used in the experiments $[10,18,20]$ was evaluated to be approximately $50 \mu \mathrm{m}$ for photon energies up to $100 \mathrm{keV}[17,19]$. For a fixed photon energy as an estimation of the spatial resolution of similar devices we may suppose size of detector pixels taking into account the root-meansquared path of the secondary electrons (photoelectrons and Auger electrons) in the detector. The path value is dependent on the energy of the detected radiation, determined according to the crystal used and the observation angle, and the working medium of the detector.

For observation angles of about $20-30^{\circ}$, as for example in Refs. $[10,18]$, and for the first reflection order, the energy of secondary electrons is approximately $10 \mathrm{keV}$ and less, which corresponds to an approximately $1 \mu \mathrm{m}$ path of the secondary electrons [31]. The typical size of a CCD pixel is approximately $10 \mu \mathrm{m}$. In particular, the pixel dimensions of the high-resolution X-ray camera are 11.6 $\times 11.2 \mu \mathrm{m}^{2}$ [17]. The dimensions of the projected colliders beam are approximately $7 \mathrm{~nm}$ and $700 \mathrm{~nm}$ (one $\sigma$ ) in vertical and horizontal directions, respectively, for ILC [1] and about $10 \mathrm{~nm}$ for CLIC [2]. These values are significantly less than $10 \mu \mathrm{m}$; therefore, direct measurement of the spatial sizes of the electron beam by transition to the X-ray range and the PXR mechanism is not feasible due to the characteristics of the existing detectors.

Therefore, we propose to measure the beam angular divergence instead of the beam size. As described in Refs. [1,2], the beam divergence is not so small (a few tens of microrads $>\gamma^{-1}$ ), although the beam size is extremely small. It is known that the main parameter that characterizes the dynamics of the particle motion in an accelerator is the beam emittance $\epsilon_{x, y}=\sigma_{x, y} \theta_{x, y}$, where $\sigma_{x, y}$ and $\theta_{x, y}$ are the respective size and divergence of the beam in the horizontal and vertical directions. Hence, measurement of the electron beam angular distribution and divergence in one plane provides information regarding the beam size in this plane from the emittance for this direction. The required value for emittance can be obtained from measurements conducted in the early stages of acceleration using conventional methods or calculation results (see, for example, Refs. [1,2]).
Conventional methods based on OTR and ODR cannot provide the required accuracy for measurement of the angular distribution of a particle accelerated to the final energy due to coherent effects. The characteristic angular size of a PXR photon beam is weakly dependent on the electron energy and can be written in the form [32]:

$\Theta_{p h}=\sqrt{\gamma^{-2}+\omega_{p}^{2} / \omega^{2}+\sigma_{m s}^{2}}$

where $\sigma_{m s}$ is the root-mean-squared angle of multiple scattering of particles in a crystal.

For observation angles of $\Theta_{D}<45^{\circ}, \Theta_{p h} \approx 2-5$ mrad, depending on the photon energy and the crystal used, exceed the divergence of the electron beam at the interaction point $\theta \approx 40$ and $15 \mu \mathrm{rad}$ in the horizontal and vertical planes, respectively, for the ILC [1], and exceed the divergence of 7-10 $\mu$ rad for the Compact Linear Collider (CLIC) [2]. Therefore, measurements of the angular distribution of PXR photons cannot help in the estimation of the electron beam divergence incident on the crystal.

PXR is always accompanied by diffracted real photons of bremsstrahlung and TR emitted in a strictly Bragg direction. The ratio between the yields of PXR photons and diffracted real photons is determined by the crystal thickness and the experimental conditions. Recently, in Ref. [25], it was reported that the ratio between the angular density of PXR intensity and DTR is significantly changed with an increase in the electron energy up to tens of giga-electron-volts and higher. The angular density of the DTR intensity becomes far higher than that for PXR.

\section{Calculation results}

As a confirmation of the above statement Fig. 1 shows the calculation results for the vertical angular distribution of radiation with the experimental geometry [18] and the first order reflections. The detailed calculation method is described in Ref. [24]. The electron beam is incident on a $50 \mu \mathrm{m}$ thick silicon crystal and the (220) reflection is investigated. The detection system is located at a distance of $1 \mathrm{~m}$ from the crystal at an angle $\Theta_{D}=2 \Theta_{B}=22.5^{\circ}$. The square detector, the size of which is $0.05 \times 0.05 \mathrm{~mm}^{2}$, is moved down through the reflex center with $0.05 \mathrm{~mm}$ steps. Curves 1 and 2 show the calculation results for the angular distributions of PXR and DTR for an electron energy of $1 \mathrm{GeV}$. Curves 3 and 4 show the same results for an electron energy of $10 \mathrm{GeV}$. The diffracted bremsstrahlung contribution is negligibly small and is not represented for conditions of $\omega=16.55 \mathrm{keV} \ll \gamma \omega_{p} \approx 60$ and $600 \mathrm{keV}$ for electron energies of 1 and $10 \mathrm{GeV}$, respectively.

Fig. 1 shows that the increase of the electron energy did not change the PXR angular distribution significantly. The small difference between the distributions for different energies is due to the lower multiple scattering for higher energy electrons. This

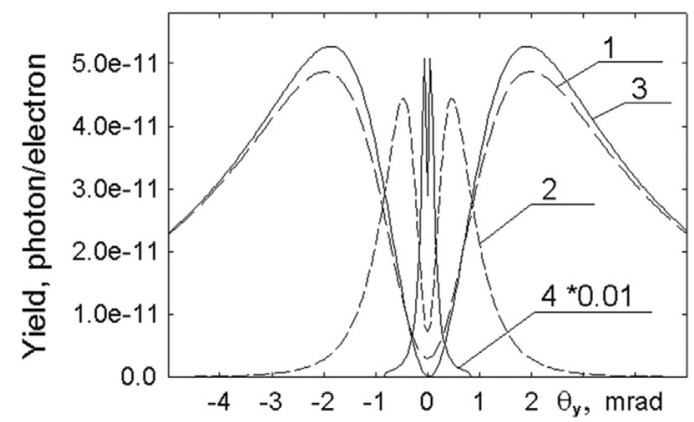

Fig. 1. Vertical distribution of the X-ray yield for the experimental geometry [18] and the first order reflection. 1 - PXR for $E_{0}=1 \mathrm{GeV} ; 2-$ DTR for $E_{0}=1 \mathrm{GeV} ; 3$ PXR for $E_{0}=10 \mathrm{GeV}$; and $4-$ DTR for $E_{0}=10 \mathrm{GeV}$. 


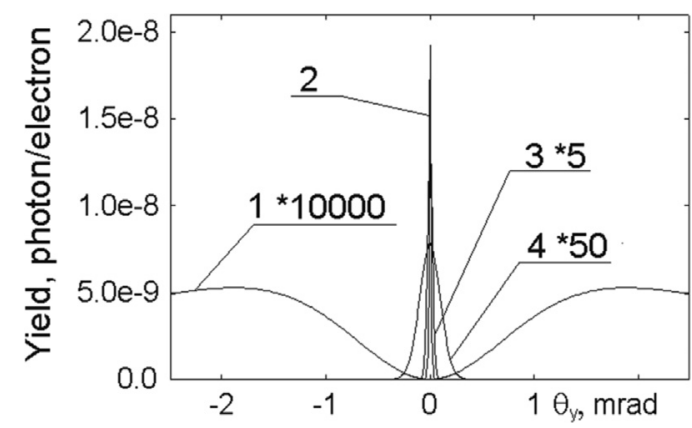

Fig. 2. Vertical distribution of the X-ray yield for an electron energy of $200 \mathrm{GeV}$ and the first order reflection. 1 - PXR; 2 - DTR for $\theta_{e} \ll \gamma^{-1} ; 3$ - DTR for $\theta_{e}=20 \mu \mathrm{rad}$; and 4 - DTR for $\theta_{e}=100 \mu \mathrm{rad}$

difference is very large for DTR angular distributions because the TR intensity is proportional to the electron energy and the characteristic size of the TR angular cone is approximately $\gamma^{-2}$; therefore, the DTR angular distribution for higher energy electrons is much narrower.

For larger electron energy and smaller electron beam divergence compared with $\Theta_{p h}$, the central part of the radiation reflex will be a low pedestal associated with the registration of PXR photons. The narrow bright peak width for $\gamma^{-1}$ that corresponds to the contribution of DTR is located in the center of the PXR angular distribution. For ILC conditions, the electron (positron) beam divergence at the interaction point $\theta_{e}$ is approximately 15$50 \mu \mathrm{rad}$ over a wide range of particle energies [1], which is significantly higher than the characteristic radiation angle $\gamma^{-1} \sim 1-$ $3 \mu \mathrm{rad}$ for particle energies above $200 \mathrm{GeV}$. Therefore, the shape of the radiation angular distribution is not dependent on the particle energy, but is defined only by the divergence of the electron (positron) beam.

To illustrate this, Fig. 2 shows the calculation results for the vertical angular distribution of the radiation for a beam of electrons with an energy of $200 \mathrm{GeV}$ incident on a $50 \mu \mathrm{m}$ thick silicon crystal, where the (220) reflection is used. The detection system is located at a distance of $2 \mathrm{~m}$ from the crystal at an angle $\Theta_{D}=2 \Theta_{B}=22.5^{\circ}$. A square detector with a size of $10 \times 10 \mu \mathrm{m}^{2}$ is moved through the reflex center with $10 \mu \mathrm{m}$ steps, which corresponds to the angular distribution measurement using an X-ray camera with the same pixel size.

Curves 1 and 2 show the calculation results of the PXR and DTR angular distribution for a point-like unidirectional beam of particles $\left(\theta_{e} \ll \gamma^{-1}\right)$. Curves 3 and 4 show that of the DTR for beam divergence of $\theta_{e}=20$ and $100 \mu \mathrm{rad}$, respectively. It is assumed that the angular distribution of the beam can be described by a twodimensional Gaussian distribution, and a standard deviation corresponds to the typical divergence angle of the beam. For simplicity, it is assumed that the values of the beam divergence in both planes are the same. With $\Theta_{p h} \gg \theta_{e}$, the PXR angular distribution for the remaining $\theta_{e}$ is virtually identical and is therefore not presented.

Fig. 2 shows that the PXR contribution is concentrated in the observation angles $\theta_{y}>0.5 \mathrm{mrad}$ and the maximum yield does not exceed $5 \times 10^{-13}$ photons/electron. At lesser angles, the PXR yield is less than $10^{-14}$ photons/electron. The DTR contribution is concentrated in the reflex center and amounts to more than the maximum PXR at 5 orders. Even for a beam divergence of $100 \mu \mathrm{rad}$, the DTR yield is 3 orders greater than the PXR yield. The intensity of higher PXR orders concentrated closer to the reflex center is substantially lower than the first order. In addition, the detection efficiencies of X-ray detectors are significantly decreased with increasing photon energy. The PXR contribution in the reflex

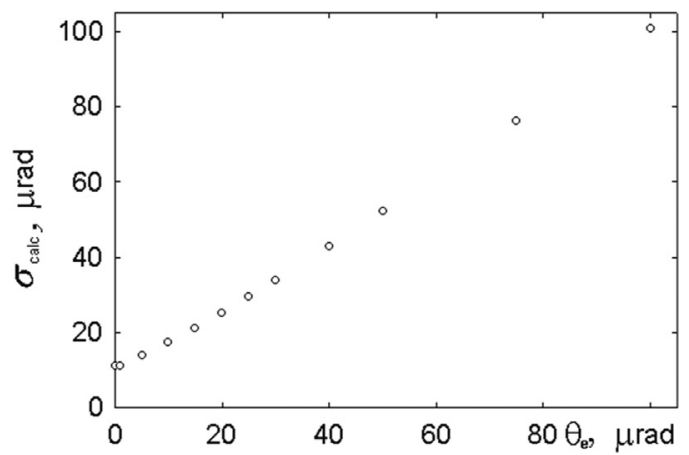

Fig. 3. Dependence of $\sigma_{\text {calc }}$ on the beam divergence $\theta_{e}$.

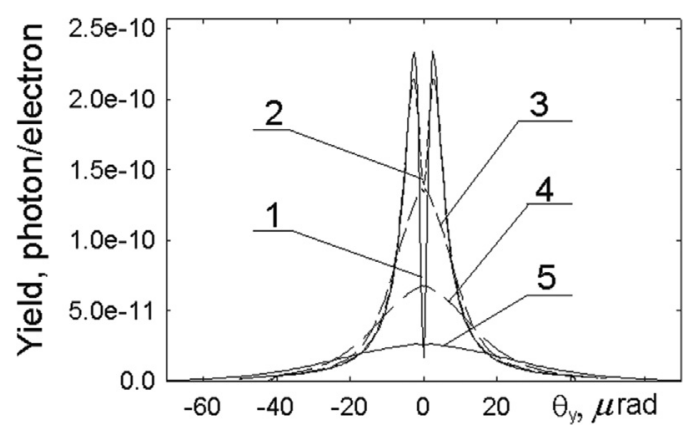

Fig. 4. Vertical distribution of the X-ray yield for an electron energy of $200 \mathrm{GeV}$ and the first order reflection. 1 - DTR for $\theta_{e} \ll \gamma^{-1} ; 2$ - DTR for $\theta_{e}=1 \mu \mathrm{rad} ; 3$ - DTR for $\theta_{e}=5 \mu \mathrm{rad} ; 4$ - DTR for $\theta_{e}=10 \mu \mathrm{rad}$; and 5 - DTR for $\theta_{e}=20 \mu \mathrm{rad}$.

center is thus negligibly small from analysis of the electron beam divergence and may be ignored.

For selected conditions where the characteristic radiation angle $\gamma^{-1} \approx 2.5 \mu \mathrm{rad}$ is less than the detector angular capture $\vartheta_{c}=5 \mu \mathrm{rad}$, the dip in the center of the DTR angular distribution is absent, and its width (see curve 2 in Fig. 2) is not more than 3-4 steps of the detector. For this reason, the difference between the angular distributions for $\theta_{e} \ll \gamma^{-1}$ and $\theta_{e}=20 \mu \mathrm{rad}$ is not very noticeable.

The dependence of $\sigma_{\text {calc }}$, which was obtained from the standard deviation in the DTR angular distribution calculated taking into account the detector angular acceptance $\left(\vartheta_{c}=5 \mu \mathrm{rad}\right)$, on the beam divergence $\theta_{e}$ is shown in Fig. 3 .

For small values of beam divergence $\left(\theta_{e}<10 \mu \mathrm{rad}\right), \sigma_{\text {calc }}$ is almost two times more than $\theta_{e}$, and the values then begin to converge. $\sigma_{\text {calc }}$ and $\theta_{e}$ practically coincide only when the condition $\theta_{e} \gg \gamma^{-1}$ is satisfied.

To explain the dependence of $\sigma_{\text {calc }}$ on the value of $\theta_{e}$, calculations of the DTR angular distributions for a crystal to detector distance of $20 \mathrm{~m}$, which corresponds to the angle of collimation $\vartheta_{c} \approx 0.5 \mu \mathrm{rad}<\gamma^{-1} \approx 2.5 \mu \mathrm{rad}$ were performed. The results of the calculations for electron beam divergence $\theta_{e}=0.2\left(\ll \gamma^{-1}\right), 1,5,10$, and $20 \mu \mathrm{rad}$ are shown by curves $1-5$ in Fig. 4, respectively, where the PXR contribution was not taken into account.

Under these conditions, the DTR angular distribution appears as expected. There is a dip in the emission intensity for the strictly Bragg direction. A proportional broadening of the angular distribution for the detected radiation is observed as $\theta_{e}$ increases. The broadening is determined by a convolution of the angular distribution of the TR and that of the electron beam incident on the crystal, and since the angular distribution of the TR has a relatively long "tail", the angular distribution of the detected radiation begins to coincide with the form of that for the electron beam only when the condition $\theta_{e} \gg \gamma^{-1}$ is satisfied. It should be remarked that 


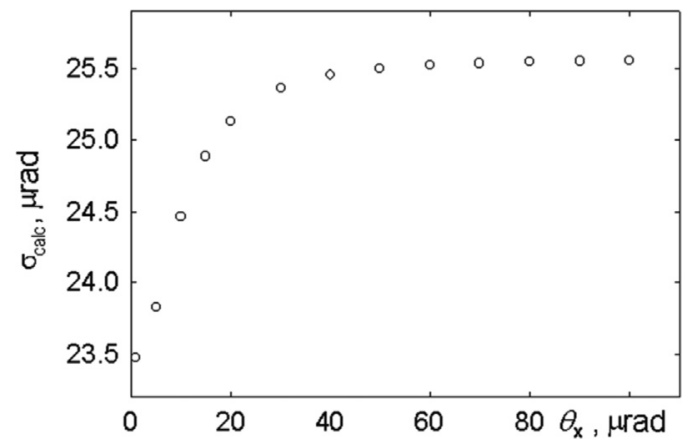

Fig. 5. Dependence of $\sigma_{\text {calc }}$ for the DTR vertical distribution on the beam divergence in the horizontal plane $\theta_{x}$.

$\sigma_{\text {calc }}$ values obtained for both distances between the crystal and the detector for the same $\theta_{e}$ appear approximately identical.

The divergence of the beam and the transverse dimensions of the ILC in the horizontal and vertical planes are substantially different. Typical values for the divergence are $\theta_{x} \sim 50 \mu \mathrm{rad}$ and $\theta_{y}$ $\sim 20 \mu \mathrm{rad}$ in the horizontal and vertical planes, respectively, [1]. Accounting for this difference will lead to some reduction in the maximum intensity of the DTR angular distribution. However, if the condition $\theta_{y} \gg \gamma^{-1}$ is satisfied, as with identical divergences in both planes, then the angular distribution of the detected radiation almost completely reproduces that of the particle beam incident on the crystal. In order to confirm this statement, we performed calculations of the DTR angular distributions under the conditions where the vertical divergence remained constant ( $20 \mu \mathrm{rad})$ while the horizontal divergence was changed from 1 to $100 \mu \mathrm{rad}$. Fig. 5 shows the dependence of $\sigma_{\text {calc }}$ for the DTR vertical distribution on the beam divergence in the horizontal plane $\theta_{x}$. The figure shows that for $\theta_{x}$ values greater than $30 \mu \mathrm{rad}$ the influence of the horizontal divergence on the shape of the DTR vertical distribution is very small (less than 1\%). For smaller horizontal divergences the $\sigma_{\text {calc }}$ value becomes smaller but the change in the $\sigma_{\text {calc }}$ value is sufficiently small (less than $9 \%$ ).

If condition $\theta_{y \gg \gamma^{-1}}$ is not satisfied, the desired value of $\theta_{e}$ can be obtained from the dispersion of the measured angular distribution and dependence shown in Fig. 3, or its analog for the two-dimensional Gaussian distribution (see Fig. 5). Thus, by measuring the DTR angular distribution, information on the electron beam divergence in both planes can be obtained, and based on the measured or calculated emittance value, the beam size at the point of the measurement can be estimated.

The main barrier to the use of DTR in thin crystals to estimate electron beam divergence and spatial sizes is the destruction of the crystal structure under the influence of the intense electron beam during the measurement process. For example, in Ref. [33] (and references therein), the degradation of coherent effects for a $0.5 \mathrm{~mm}$ thick diamond crystal was observed at an electron energy of $16 \mathrm{GeV}$ and a beam density of approximately $10^{19}$ electrons per $\mathrm{cm}^{2}$. For the ILC with a spatial beam size of $\sim 700 \times 7 \mathrm{~nm}^{2}$, the beam density for one spill $\left(N_{e} \approx 2.6 \times 10^{13}\right.$ electrons per spill) will be greater than the critical density for a $0.5 \mathrm{~mm}$ thick diamond crystal by several orders. For a thinner crystal, a similar effect will be observed for a greater total beam density; however, crystal degradation will occur much faster.

A possible solution to the crystal destruction problem may be the use of surface PXR (see Ref. [34] and references therein). This effect is related with the excitation of electrons in crystal atoms by the electromagnetic field of a charged particle moving near the crystal surface and its subsequent irradiation. To realize this phenomenon, it is necessary that the distance between the particle and the crystal surface is approximately $\gamma \lambda$ or less, where $\lambda$ is the wavelength of the emission measured. The intensity of the radiation is close to the PXR intensity with a correction factor of approximately $\exp (-r / \gamma \lambda)$, where $r$ is the distance between the particle trajectory and the crystal surface.

Surface PXR is similar to the Smith-Purcell effect [35], which is already used for electron beam parameter diagnostics in accelerator physics [5]. In both cases, the interference of electron radiation from atoms under the field influence of a charged moving particle is measured. However, the X-ray radiation wavelength for the experimental observation of this emission mechanism is small and has thus not been discussed yet. For the ILC and CLIC conditions, $\gamma \lambda$ becomes sub-millimeter; therefore, this type of radiation can be experimentally observed and used for electron beam parameter diagnostics.

However, here we have the same problem as with typical PXR in crystals. The characteristic angular size of the PXR photon beam is far larger than the beam divergence measured. From the analogy between the surface and typical PXR, we may expect surface diffracted transition radiation with parameters close to those of typical DTR. If this type of emission does exist, then we may expect that it could be used for the measurement of high-energy electron beam parameters. As confirmation for the existence of surface diffracted transition radiation, we may consider the experimental results [36], where under Smith-Purcell radiation measurements bright emission under a small angle to the direction of electron movement was observed. This radiation may be interpreted as transition radiation arising under particle movement near an extended target without its intersection.

\section{Summary and conclusions}

The results of the study may be briefly stated as follows:

(1) The space resolution of any devices for X-ray beam spatial distribution measurements is limited by the size of the CCD pixel. The sizes of the planned electron-positron linear collider beams in both directions are far less than the typical size of a CCD pixel or other devices used for X-ray spatial distribution measurements. Therefore, PXR spatial distribution measurements cannot provide information regarding the sizes of such beams.

(2) These data may be obtained from information on electron beam emittance in both planes and divergence, which may be obtained from measurements of an electron beam angular distribution. The beam emittance may be obtained from calculations or measurements at the earlier stages of acceleration using traditional methods with optical devices.

(3) For a particle energy approximately some hundreds of gigaelectron-volts, the DTR intensity in a narrow cone is far larger than the PXR intensity. The DTR angular distribution is compared with the electron beam angular distribution and may be used for measurement of the electron beam divergence.

(4) In contrast to the Shintake monitor, the devices used in the proposed method are less expensive and may be installed in any part of the accelerator to control the electron beam parameters in the acceleration process.

(5) The main barrier that can prevent the use of DTR in thin crystals for the estimation of electron beam divergence and spatial sizes is destruction of the crystal structure under the influence of a high-intensity beam. For the ILC and CLIC conditions, crystal structure degradation can be expected after one spill of the accelerator. However, the proposed method may prove useful when the beam current is low, such as at the commissioning stage of the accelerator.

(6) A possible solution to the crystal destruction problem may be the use of surface PXR and DTR. These types of fast electron 
emission in crystals were not observed yet. However experimental verification of these effects may help us in the estimation of the collider beam parameters. It requires both additional experimentation and theoretical investigations, which will be presented elsewhere.

\section{Acknowledgments}

The authors wish to thank previous co-authors [21,24] for their help in the development and implementation of the methodologies used in the research process. This work was supported by a grant from the Russian Science Foundation (Project N 15-1210019) and in part by a Kakenhi Grant-in-Aid (No. 26400304) from the Japan Society for the Promotion of Science (JSPS).

\section{References}

[1] ILC Technical Design Report, 2013.

[2] A Multi-TeV Linear Collider Based on CLIC Technology: CLIC Conceptual Design Report, 2012.

[3] R.B. Fiorito, in: Proceedings of PAC09, 2009, p. 741.

[4] J. Urakawa, H. Hayano, K. Kubo, S. Kuroda, N. Terunuma, M. Kuriki, T. Okugi, T. Naito, S. Araki, A. Potylitsyn, G. Naumenko, P. Karataev, N. Potylitsyna, I. Vnukov, T. Hirose, R. Hamatsu, T. Muto, M. Ikezawa, Y. Shibata, Nuclear Instruments and Methods in Physics Research Section A 472 (2001) 309.

[5] G. Kube, H. Backe, W. Lauth, H. Schöpe, in: Proceedings of DIPAC2003, 2003, p. 40.

[6] H. Loos et al., in: Proceedings of FEL08, 2008, p. 485.

[7] T. Shintake, Nuclear Instruments and Methods in Physics Research Section A 311 (1992) 453

[8] J. Yan, Y. Yamaguchi, Y. Kamiya, S. Komamiya, M. Oroku, T. Okugi, N. Terunuma, K. Kubo, T. Tauchi, J. Urakawa, Nuclear Instruments and Methods in Physics Research Section A 740 (2014) 131.

[9] A. Gogolev, A. Potylitsyn, G. Kube, Journal of Physics: Conference Series 357 2012) 012018.

[10] Y. Takabayashi, Physics Letters A 376 (2012) 2408.

[11] G.M. Garibyan, C. Yang, Soviet Physics JETP 34 (1972) 495.

[12] V.G. Baryshevsky, I.D. Feranchuk, Soviet Physics JETP 34 (1972) 502.

[13] V.P. Lapko, N.N. Nanosov, Soviet Physics-Technical Physics 35 (5) (1990) 633.

[14] N.N. Nasonov, Nuclear Instruments and Methods in Physics Research Section B 145 (1998) 19.
[15] H. Nitta, Nuclear Instruments and Methods in Physics Research Section B 115 (1996) 401.

[16] K.-H. Brenzinger, B. Limburg, H. Backe, S. Dambach, H. Euteneuer, F. Hagenbuck, C. Herberg, K.H. Kaiser, O. Kettig, G. Kube, W. Lauth, H. Schöpe, Th. Walcher, Physical Review Letters 79 (1997) 2462.

[17] High-Resolution X-Ray Camera 〈http://www.proxivision.de/datasheets/X-RayCamera-HR25-X-ray-PR-0055E-03.pdf〉.

[18] G. Kube, C. Behrens, A.S. Gogolev, Yu.P. Popov, A.P. Potylitsyn, W. Lauth, S. Weisse, in: Proceedings of IPAC2013, 2013, p. 491.

[19] A.L. Meadowcroft, C.D. Bentley, E.N. Stott, Review of Scientific Instruments 79 (2008) 113102.

[20] Y. Takabayashi, K. Sumitani, Physics Letters A 377 (2013) 2577.

[21] A.N. Baldin, I.E. Vnukov, B.N. Kalinin, E.A. Karataeva, Poverkhnost' 4 (2006) 72 (in Russian)

[22] K.-H. Brenzinger, C. Herberg, B. Limburg, H. Backe, S. Dambach, H. Euteneuer F. Hagenbuck, H. Hartmann, K. Johann, K.H. Kaiser, O. Kettig, G. Knies, G. Kube, W. Lauth, H. Schöpe, Th. Walcher, Zeitschrift für Physik A 358 (1997) 107.

[23] Y. Takabayashi, A.V. Shchagin, Nuclear Instruments and Methods in Physics Research Section B 278 (2012) 78.

[24] S.A. Laktionova, O.O. Pligina, M.A. Sidnin, I.E. Vnukov, Journal of Physics: Conference Series 517 (2014) 012020.

[25] Yu.A. Goponov, S.A. Laktionova, O.O. Pligina, M.A. Sidnin, I.E. Vnukov, Nuclear Instruments and Methods in Physics Research Section B 355 (2015) 150.

[26] H. Nitta, Physics Letters A 158 (1991) 270.

[27] E.A. Bogomazova, B.N. Kalinin, G.A. Naumenko, D.V. Padalko, A.P. Potylitsyn, A. F. Sharafutdinov, I.E. Vnukov, Nuclear Instruments and Methods in Physics Research Section B 201 (2003) 276.

[28] M.L. Ter-Mikaelian, High-Energy Electromagnetic Processes in Condensed Media, Wiley-Interscience, New York, 1972.

[29] P.L. Anthony, R. Becker-Szendy, P.E. Bosted, M. Cavalli-Sforza, L.P. Keller, L A. Kelley, S.R. Klein, G. Niemi, M.L. Perl, L.S. Rochester, J.L. White, Physical Review Letters 76 (1996) 3550.

[30] V.A. Bazylev, N.K. Zhevago, Radiation of Relativistic Particles in External Fields and in Matter, Nauka Pub., Moscow, 1987 (in Russian).

[31] V.I. Bespalov, Vzaimodeistvie ioniziruyushchikx izluchenii s veshchestvom, Tomsk, TPU, 2008, p. 396 (in Russian).

[32] I.D. Feranchuk, A.V. Ivashin, Journal of Physics (Paris) 46 (1985) 1981.

[33] R. Schwitters, The SLAC Coherent Bremsstrahlung Facility, SLAC-TN-70-32, 1970.

[34] A.I. Benediktovitch, I.D. Feranchuk, Journal of Physics: Conference Series 236 (2010) 012015.

[35] S.J. Smith, E.M. Purcell, Physical Review 92 (1953) 1069.

[36] B.N. Kalinin, D.V. Karlovets, A.S. Kostousov, G.A. Naumenko, A.P. Potylitsyn, G. A. Saruev, L.G. Sukhikh, Nuclear Instruments and Methods in Physics Research Section B 252 (2006) 62. 